\title{
Vitamin D supplementation in pregnancy: How much is optimal?
}

\author{
Salima Kerai
}

Research Specialist, Peadiatrics and Child Health, Aga Khan University, Karachi, Pakistan.

Pregnancy is a crucial time period in the life of a woman where her wellbeing goes beyond her health to include the wellbeing of her developing fetus. The therapeutic role of vitamin D during pregnancy is under research. Vitamin D has captured the attention of scientists in its role beyond calcium and bone metabolism. Some of the effects of this important vitamin transcend from its traditional role in bone mineralization to protecting mother from adverse health conditions and strengthening the immune system in neonates and infants.

Vitamin D status is commonly assessed through measuring serum levels of 25-hydroxyvitamin D [25 $(\mathrm{OH}) \mathrm{D}]$ which is a stable metabolite of vitamin $\mathrm{D}$. It is converted into its active metabolite 1,25dihydroxyvitamin $\mathrm{D}\left[1,25(\mathrm{OH})_{2} \mathrm{D}\right]$ after cutaneous production or absorption from food or supplements. There is an ongoing debate about optimal serum levels of this vitamin for achieving favorable health outcomes. While, low levels of this important vitamin in pregnancy (also referred as maternal hypovitaminosis defined as serum level $<20 \mathrm{ng} / \mathrm{ml}$ or $<100 \mathrm{nmol} / \mathrm{L}$ ) is considered as a significant risk factor for adverse maternal conditions including preeclampsia and gestational diabetes. ${ }^{1}$ Epidemiological studies on vitamin D status in this high risk population in Pakistan suggest degrees of deficiency. Serum level and nutrient intake study among women residing in different towns of Karachi showed that $91.5 \%$ of women are deficient in Vitamin D. ${ }^{2} 73 \%$ deficieincy is reported from a crosssectional survey among women of child bearing age from Lahore, Pakistan. ${ }^{3}$ In a major teaching hospital in Karachi, Atiq et al found $55 \%$ of healthy breast-fed infants and $45 \%$ of nursing mothers have low serum vitamin D levels. ${ }^{4}$ The findings above reflect a widespread deficiency of vitamin D among women of child bearing age and their offspring in Pakistani society. Without adequate nutrient intake and sunlight exposure, vitamin $\mathrm{D}$ supplementation becomes necessary. There is inconclusive evidence related to harm or benefits of vitamin D supplementation during pregnancy. World Health Organization (WHO) does not recommend the routine use of this vitamin during pregnancy. Furthermore, current recommended daily allowance (RDA) is considered inadequate to attain optimal production of vitamin $\mathrm{D}$ during pregnancy. ${ }^{5}$

Evidence shows that Vitamin D not only reduces the risk of preeclampsia, preterm birth and low birth weight but it also helps in preventing dental caries, respiratory infections, sepsis and asthma in infancy. ${ }^{6,7}$ Although, the underlying mechanism of action of this important vitamin in above mentioned conditions is yet to be known. The evidence on whether a routine vitamin D supplementation and at what dose should be given as a part of routine antenatal care to all women is still under research. This paper examines a few experimental studies comparing various doses of vitamin D supplementation during pregnancy and its impact on maternal and neonatal outcomes.

In a randomized controlled trial (RCT) recruiting women with a singleton pregnancy from a university hospital at South Carolina at 12-16 weeks gestation, randomized women to receive 400,2000 or 4000 international units (IU) of vitamin $\mathrm{D}_{3}$ /day until delivery. Vitamin D supplementation of 4000 IU/day was found to be safe and most effective in achieving sufficiency in all women and their neonates. Mothers had significantly fewer pregnancy complications, including small for gestational age at birth. ${ }^{8}$ In another multicenter trial from three sites in UK (Southampton, Oxford, and Sheffield) titled 'Maternal Vitamin D Osteoporosis Study' (MAVIDOS) recruited pregnant women with a singleton pregnancy, and a serum vitamin D concentration of $25-100 \mathrm{nmol} / \mathrm{L}$ at $10-17$ weeks of gestation. Participants were randomly assigned to either vitamin $D_{3} 1000 \mathrm{IU} /$ day or matched placebo, taken orally, for 14 weeks until delivery. The study showed that supplementation with vitamin $\mathrm{D}_{3} 1000 \mathrm{IU} /$ day during pregnancy did not lead to increased offspring wholebody bone mineral content compared to placebo. However, it established that 1000 IU of vitamin D is safe during pregnancy. ${ }^{9}$ These studies from western part of the world appear to be inconclusive regarding the role and dose of vitamin D in improving health of mother and her child. While both $1000 \mathrm{IU}$ and $4000 \mathrm{IU}$ of vitamin D per day appeared to be safe in pregnancy.

There are a handful of studies from South Asian region of confirming the role of vitamin D in improving health of woman and her offspring but lacks comparability and validity of findings are unclear. In a study in India, pregnant mothers were randomized in the second trimester to receive either one oral dose of $60,000 \mathrm{IU}$ of vitamin $\mathrm{D}_{3}$ or two doses of 120,000 IU of vitamin $\mathrm{D}_{3}$ each in the second and third trimesters. Both doses found to have a beneficial effect on infant anthropometry, the larger dose also shown to improve cord blood alkaline phosphatase and maternal vitamin $\mathrm{D}$ serum levels. ${ }^{10}$ From the same country another open label

Correspondence: Salima Kerai, Research Specialist, Peadiatrics and Child Health, Aga Khan University, Karachi, Pakistan. E-mail: Salima.kerai@aku.edu. 
RCT showed that $2000 \mathrm{IU} /$ day is safe and effective as compared to $1000 \mathrm{IU} /$ day in achieving Vitamin D sufficiency in pregnant women. ${ }^{11}$

Another study in Bangladesh used a supplementation regimen consisting of $5000 \mathrm{IU}$ of vitamin $\mathrm{D}_{3} /$ day over the median period of 10 weeks beginning from week 26 -30 of pregnancy. Vitamin $\mathrm{D}_{3}$ supplementation $(35,000 \mathrm{IU} /$ week $)$ significantly rose maternal and neonatal (cord blood) serum levels of vitamin D concentrations and infants of supplemented mother had a significantly higher length-for-age $z$-score at 1 year of age. ${ }^{12}$ Another trial from Iran showed that 50,000 IU of vitamin D supplementation every 2 weeks versus 400 IU vitamin D daily decreased the incidence of gestational diabetes. However, there were no differences in the incidence of preeclampsia, gestational hypertension, preterm labor, and low birth weight between two groups. ${ }^{13}$ Open label trial in a university hospital in Karachi, Pakistan compared $200 \mathrm{mg}$ of ferrous sulfate and $600 \mathrm{mg}$ of calcium versus $4000 \mathrm{IU}$ of vitamin $D_{3}$ daily to see its effects on obstetric and neonatal outcomes. Results showed that maternal and neonatal vitamin D levels were significantly higher in the group that received vitamin D supplementation with better one and five minute Apgar scores for neonates at birth. ${ }^{14}$ The data presented above seems to be inconclusive and incomparable for the optimal and safe dosing of supplementation of this key vitamin during pregnancy from this region. Therefore, further high quality trials are needed to confirm the safety and therapeutic dose.

Available observational data suggest that the function of vitamin $\mathrm{D}$ extends beyond calcium hemeostasis during pregnancy. Research has shown that the potential role of vitamin $\mathrm{D}$ in pregnancy in the prevention of adverse maternal and neonatal conditions. However, deductions cannot be made from experimental studies as they lack comparability in regimen of vitamin D supplementation and rigor in their design and trial conduct. Therefore, evidence on dose of routine vitamin D supplementation in pregnancy from these randomized controlled trials can be considered as inconclusive. While it is accepted that supplementation is not harmful, further rigorous randomized controlled trials are required to confirm these effects with optimal dosage.

\section{References}

1. Wagner CL, Hollis BW, Kotsa K, Fakhoury H, Karras SN. Vitamin D administration during pregnancy as prevention for pregnancy, neonatal and postnatal complications. Rev Endocr Metab Disord 2017:1-16.

2. Khan AH, Iqbal R, Naureen G, Dar FJ, Ahmed FN. Prevalence of vitamin D deficiency and its correlates: results of a community-based study conducted in Karachi, Pakistan. Arch Osteoporos 2012; 7:275-82.

3. Junaid K, Rehman A, Jolliffe DA, Wood K, Martineau AR. High prevalence of vitamin D deficiency among women of child-bearing age in Lahore Pakistan, associating with lack of sun exposure and illiteracy. BMC Womens Health 2015;15:83.

4. Atiq M, Suria A, Nizami SQ, Ahmed I. Vitamin D status of breastfed Pakistani infants. Acta Paediatr 1998;87(7):737-40.

5. Wagner CL, Taylor SN, Johnson DD, Hollis BW. The role of vitamin D in pregnancy and lactation: emerging concepts. Womens Health 2012;8(3):32340.

6. Karras SN, Fakhoury H, Muscogiuri G, Grant WB, van den Ouweland JM, Colao AM, et al. Maternal vitamin D levels during pregnancy and neonatal health: evidence to date and clinical implications. Ther Adv Musculoskelet Dis 2016;8(4):124-35.

7. De-Regil LM, Palacios C, Lombardo LK, Pena-Rosas JP. Vitamin D supplementation for women during pregnancy. São Paulo Med J 2016;134(3):274-5.

8. Hollis BW, Johnson D, Hulsey TC, Ebeling M, Wagner CL. Vitamin D supplementation during pregnancy: double-blind, randomized clinical trial of safety and effectiveness. J Bone Miner Res 2011;26(10):2341-57.

9. Cooper C, Harvey NC, Bishop NJ, Kennedy S, Papageorghiou AT, Schoenmakers I, et al. Maternal gestational vitamin D supplementation and offspring bone health (MAVIDOS): a multicentre, double-blind, randomized placebo-controlled trial. Lancet Diabetes Endocrinol 2016;4(5):393-402.

10. Kalra P, Das V, Agarwal A, Kumar M, Ramesh V, Bhatia $\mathrm{E}$, et al. Effect of vitamin D supplementation during pregnancy on neonatal mineral homeostasis and anthropometry of the newborn and infant. $\mathrm{Br} \mathrm{J}$ Nutr 2012;108(6):1052-8.

11. Mir SA, Masoodi SR, Shafi S, Hameed I, Dar MA, Bashir MI, et al. Efficacy and safety of Vitamin D supplementation during pregnancy: A randomized trial of two different levels of dosing on maternal and neonatal Vitamin D outcome. Indian $J$ Endocrinol Metab 2016;20(3):337-42.

12. Roth DE, Perumal N, Al Mahmud A, Baqui AH. Maternal vitamin D3 supplementation during the third trimester of pregnancy: effects on infant growth in a longitudinal follow-up study in Bangladesh. J Pediatr 2013;163(6):1605-11.

13. Mojibian M, Soheilykhah S, Fallah Zadeh MA, Jannati Moghadam M. The effects of vitamin D supplementation on maternal and neonatal outcome: A randomized clinical trial. Iran $J$ Reprod Med 2015;13(11):687-96.

14. Hossain N, Kanani FH, Ramzan S, Kausar R, Ayaz $\mathrm{S}$, Khanani $\mathrm{R}$, et al. Obstetric and neonatal outcomes of maternal vitamin D supplementation: results of an open-label, randomized controlled trial of antenatal vitamin D supplementation in Pakistani women. J Clin Endocrinol Metab 2014;99(7):244855 . 